\title{
Linked decreases in liver kinase B1 and AMP-activated protein kinase activity modulate matrix catabolic responses to biomechanical injury in chondrocytes
}

Freyr Petursson ${ }^{1}$, Matt Husa ${ }^{2}$, Ron June ${ }^{3}$, Martin Lotz ${ }^{4}$, Robert Terkeltaub ${ }^{1 *}$ and Ru Liu-Bryan ${ }^{1 *}$

\begin{abstract}
Introduction: AMP-activated protein kinase (AMPK) maintains cultured chondrocyte matrix homeostasis in response to inflammatory cytokines. AMPK activity is decreased in human knee osteoarthritis (OA) chondrocytes. Liver kinase B1 (LKB1) is one of the upstream activators of AMPK. Hence, we examined the relationship between LKB1 and AMPK activity in OA and aging cartilages, and in chondrocytes subjected to inflammatory cytokine treatment and biomechanical compression injury, and performed translational studies of AMPK pharmacologic activation.
\end{abstract}

Methods: We assessed activity (phosphorylation) of LKB1 and AMPKo in mouse knee OA cartilage, in aging mouse cartilage (6 to 24 months), and in chondrocytes after mechanical injury by dynamic compression, via immunohistochemistry or western blot. We knocked down LKB1 by siRNA transfection. Nitric oxide, matrix metalloproteinase (MMP)-3, and MMP-13 release were measured by Griess reaction and ELISA, respectively.

Results: Knockdown of LKB1 attenuated chondrocyte AMPK activity, and increased nitric oxide, MMP-3 and MMP13 release $(P<0.05)$ in response to $I L-1 \beta$ and TNF $\alpha$. Both LKB1 and AMPK activity were decreased in mouse knee $\mathrm{OA}$ and aged knee cartilage, and in bovine chondrocytes after biomechanical injury. Pretreatment of bovine chondrocytes with AMPK activators AICAR and A-769662 inhibited both AMPK $\alpha$ dephosphorylation and catabolic responses after biomechanical injury.

Conclusion: LKB1 is required for chondrocyte AMPK activity, thereby inhibiting matrix catabolic responses to inflammatory cytokines. Concurrent loss of LKB1 and AMPK activity in articular chondrocytes is associated with OA, aging and biomechanical injury. Conversely, pharmacologic AMPK activation attenuates catabolic responses to biomechanical injury, suggesting a potentially novel approach to inhibit OA development and progression.

Keywords: osteoarthritis, cartilage, aging, MMP-3, nitric oxide

\section{Introduction}

Osteoarthritis (OA) is a disorder of the synovial joint, which culminates in articular cartilage degeneration, and associated pain and disability [1,2]. Age and biomechanical injury are implicated as primary risk factors for OA $[1,2]$. Chondrocytes, the sole cells residing within articular

\footnotetext{
* Correspondence: rterkeltaub@ucsd.edu; ruliu@ucsd.edu

${ }^{1}$ VA San Diego Healthcare System, Department of Medicine, University of California San Diego, 111K, 3350 La Jolla Village Drive, San Diego, CA 92161, USA

Full list of author information is available at the end of the article
}

cartilage, are responsible for maintaining the homeostatic balance between matrix anabolism and catabolism [1,2]. However, biomechanical injury initiates a sequence of biological events in the joint, in which chondrocyte dysfunction and loss of viability lead to progressive articular cartilage damage [3]. In addition, aged chondrocytes exhibit an impaired ability to respond to mechanical and inflammatory insults, manifested as decreased anabolic activity and an increase in catabolic activity, thereby compromising cartilage extracellular matrix integrity $[4,5]$.

\section{() Biomed Central}

(c) 2013 Petursson et al.; licensee BioMed Central Ltd. This is an open access article distributed under the terms of the Creative Commons Attribution License (http://creativecommons.org/licenses/by/2.0), which permits unrestricted use, distribution, and reproduction in any medium, provided the original work is properly cited. 
IL-1 $\beta$, TNF $\alpha$, and other inflammatory mediators in injured and aging joints [6-9] play a significant role in promoting catabolism of type II collagen and proteoglycans [2]. The serine/threonine protein kinase AMP-activated protein kinase (AMPK), a fuel-sensing, master regulator of energy homeostasis and cellular metabolism $[10,11]$, exerts anti-inflammatory effects, partly mediated by inhibition of NF- $\kappa$ B signaling [12]. We demonstrated that AMPK activity is constitutively present in normal articular chondrocytes, but is decreased in human knee OA chondrocytes [13]. IL-1 $\beta$ and TNF $\alpha$ induce marked loss of AMPK activity in normal articular chondrocytes [13]. Conversely, AMPK pharmacological activators attenuate cartilage explant and monolayer cultured chondrocyte procatabolic responses to IL-1 $\beta$ and TNF $\alpha$ [13]. Hence, decreased AMPK activity in articular chondrocytes has the potential to disrupt cartilage homeostasis by promoting matrix catabolism, thereby contributing to progression of OA.

AMPK activation is induced by several upstream kinases via AMPK $\alpha$ subunit phosphorylation at a conserved threonine; dephosphorylation by protein phosphatases inactivates AMPK [10,11]. Liver kinase B1 (LKB1), a serine/ threonine protein kinase that was first identified as a tumor suppressor, is one of the upstream kinases that activate AMPK $[10,11]$. Here, we determined the relationship between LKB1 and AMPK activities in cultured chondrocytes, and examined phosphorylation of LKB1 and $\mathrm{AMPK} \alpha$ in human knee OA chondrocytes, in mouse knee $\mathrm{OA}$ and aging cartilages, and in bovine knee chondrocytes embedded in alginate after biomechanical injury. Our results closely link matrix catabolism with decreased LKB1 and $\mathrm{AMPK}$ activities, present in $\mathrm{OA}$, aging, and injured chondrocytes. Conversely, we establish that AMPK pharmacologic activators inhibit catabolic responses following biomechanical injury in chondrocytes.

\section{Materials and methods Reagents}

All chemical reagents were obtained from Sigma-Aldrich (St Louis, MO, USA), unless otherwise indicated. AMPK pharmacologic activators 5-aminoimidazole-4-carboxyamide ribonucleoside (AICAR) and A-769662 were from Tocris Bioscience (Bristol, UK). Recombinant human IL$1 \beta$ and TNF $\alpha$, and matrix metalloproteinase (MMP)-3 and MMP-13 ELISA kits were purchased from R\&D Systems, Inc. (Minneapolis, MN, USA). Antibodies to phospho-LKB1 (Ser428), phospho-AMPK $\alpha$ (Thr172), total AMPK $\alpha$ and cleaved caspase-3 were from Cell Signaling Technology, Inc. (Danvers, MA, USA) for western blot, and from Abcam (Cambridge, MA, USA) for immunohistochemistry. Human LKB1 siRNA and control siRNA were from Santa Cruz Biotechnology (Santa Cruz, CA, USA).

\section{Studies of human knee articular chondrocytes}

Studies were performed in compliance with an institutionally reviewed and approved human subject protocol by the IRB at the Scripps Research Institute (La Jolla, CA, USA). The human knee chondrocytes were isolated from autopsy donors (no consent was needed) that were graded macroscopically according to a modified Outerbridge scale $[14,15]$. Grade I represents intact cartilage surface (normal); grade II represents minimal fibrillation (OA); and grade III represents overt fibrillation (OA) $[14,15]$. Human chondrocytes were cultured in high-glucose DMEM with $10 \% \mathrm{FCS}, 100 \mu \mathrm{g} / \mathrm{ml}$ streptomycin, and $100 \mathrm{IU} / \mathrm{ml}$ penicillin at $37^{\circ} \mathrm{C}$, and no later than first passage chondrocytes were used for all experiments. Unless otherwise indicated, chondrocytes were plated at $2.5 \times 10^{5}$ cells per well in $250 \mu \mathrm{l}$ medium on the day before the experimental treatment in 12-well plates.

\section{Experimental osteoarthritis models in mice}

All mouse experiments were performed in compliance with an institutionally reviewed and approved protocol by IACUC at the Scripps Research Institute. Joint instabilityinduced OA was induced in 2-month-old C57BL/6J mice by transection of the anteromedial meniscotibial ligament and the medial collateral ligament, and animals were euthanized 8 weeks later. Aging C57BL/6 mice were kept under normal conditions and knee joints were compared at 6, 12 and 24 months of age. Knee joints from surgical $\mathrm{OA}$ and aging mice groups were resected, fixed in $10 \%$ zinc-buffered formalin (Z-Fix; Anatech, Battle Creek, MI, USA) for 2 days, decalcified in TBD-2 (Shandon, Pittsburgh, PA, USA) for 72 hours, and paraffin embedded using standard protocols.

\section{Immunohistochemistry}

Mouse knee cartilage sections were pretreated with hyaluronidase $(2 \mathrm{mg} / \mathrm{ml})$ for 1 hour before being treated with $3 \%$ (vol/vol) $\mathrm{H}_{2} \mathrm{O}_{2}$ for 10 minutes. The sections were then blocked with $10 \%$ goat serum for 2 hours at room temperature. After washing with Tris-buffered saline, rabbit antibodies to phospho-LKB1 (Ser428; 1:50 dilution), phospho-AMPK $\alpha$ (Thr172; 1:50 dilution) and the negative control rabbit IgG $(1 \mu \mathrm{g} / \mathrm{ml})$ were applied to the sections and incubated overnight at $4{ }^{\circ} \mathrm{C}$. Next, the sections were washed with Tris-buffered saline, incubated with biotinylated goat anti-rabbit IgG secondary antibody for 30 minutes, and then incubated for 30 minutes using the Histostain Plus kit (Invitrogen, Carlsbad, CA, USA). Finally, the sections were washed and incubated with 3,3'-diaminobenzidine substrate for 2 to 5 minutes.

\section{Quantification of positive staining chondrocytes}

Positive staining cells in the noncalcified region of femoral and tibial cartilage of each mouse knee section 
were counted from six different areas of the knee (representing the center of the femoral condyle and tibia that are not covered by the menisci as well as the medial and lateral femoral condyles and tibia) adapted from the previous method [16]. The cellularity was quantified by counting the number of cells stained with hematoxylin on corresponding, adjacent sections. The number of positive cells for each antibody was expressed as the percentage of positive staining cells via immunohistochemistry, relative to the number of cells stained with hematoxylin in corresponding sections.

\section{Knockdown of LKB1 in human knee articular chondrocytes}

Normal cultured primary human knee articular chondrocytes (passage 1) were transfected with siRNAs of LKB1 and nontarget control. Transfection used the Amaxa Nucleofection ${ }^{\mathrm{TM}}$ system (Amaxa Inc., Gaithersburg, MD, USA), according to the manufacturer's protocol. Knockdown of expression of LKB1 was examined by SDS-PAGE/western blot analysis.

\section{Bovine knee chondrocytes subjected to biomechanical injury}

Bovine articular chondrocytes, isolated from mature cow knees as described previously [17], were embedded in $2 \%$ alginate discs with $6 \mathrm{~mm}$ diameter and $3 \mathrm{~mm}$ height. The chondrocyte-alginate constructs were then cultured in DMEM medium, containing $10 \%$ FBS and $1 \%$ penicillinstreptomycin, in a $37^{\circ} \mathrm{C}, 5 \% \mathrm{CO}_{2}$ incubator for 3 to 4 weeks. This time period was based on prior knowledge that chondrocytes embedded in alginate require approximately 3 weeks to establish a consistent pericellular and territorial extracellular matrix [18]. This approach allowed for robust extracellular matrix production before application of biomechanical injury, which used a custom-made mechanical compression apparatus housed inside a $37^{\circ} \mathrm{C}, 5 \% \mathrm{CO}_{2}$ incubator. Sub-lethal injury was optimized to induce no significant increase cell death at a time point immediately after completion of compression (relative to no compression), with cell viability determined in situ by the Live/Dead cell viability assay (Invitrogen). The sub-lethal injury condition (defined as no immediate cell death), was associated with delayed cell death (to about $25 \%$ of cells) after injury. The conditions involved continuous dynamic unconfined compression at $24 \%$ strain, with $12 \%$ amplitude at $0.5 \mathrm{~Hz}$ for 16 hours. The alginate-chondrocyte constructs were then cultured and analyzed at 0, 1, 2 and 5 days after injury. Chondrocytes were isolated from the constructs by dissolution of alginate using a $50 \mathrm{mM}$ ethylenediamine tetraacetic acid/ PBS solution, and the cell lysates were subjected to SDSPAGE/western blot analysis for expression of cleaved caspase-3, an apoptosis marker, and phosphorylation of
LKB1 and AMPK $\alpha$. Conditioned media were also collected for measurement of release of nitric oxide (NO) and glycosaminoglycans (GAGs) [13]. Expression of MMP-3 was determined at both the mRNA level by quantitative RT-PCR analysis and the protein level by SDS-PAGE/western blot analysis of the conditioned media.

\section{Statistical analyses}

All data were uniformly expressed as the mean \pm standard deviation. Statistical analyses were performed by two-way analysis of variance with Bonferroni post-hoc test using GraphPad PRISM 5 (GraphPad, La Jolla, CA, USA). $P<0.05$ was considered significant.

\section{Results}

Inhibition of phosphorylation of AMPK $\alpha$ and enhanced catabolic responses to IL-1 $\beta$ and TNF $\alpha$ via LKB1 knockdown in chondrocytes

To determine whether LKB1 regulates AMPK activity in chondrocytes, we knocked down LKB1 expression in normal cultured human knee articular chondrocytes (passage 1) via transfection with siRNAs of LKB1 and the nontarget control. The significant decrease in LKB1 protein expression by siRNA was confirmed by western blot (Figure 1A). Next, we examined phosphorylation of AMPK $\alpha$ in the LKB1 knockdown chondrocytes and in the control cells in the presence or absence of IL- $1 \beta$ and TNF $\alpha$. As with our previous observation [13], phosphorylation of AMPK $\alpha$ was constitutively present but was decreased by IL-1 $\beta$ and TNF $\alpha$ in the control cells (Figure 1B). In comparison, phosphorylation of AMPK $\alpha$ was almost completely inhibited at the basal level and was blunted by IL-1 $\beta$ and TNF $\alpha$ in the LKB1 knockdown chondrocytes (Figure 1B), indicative of a necessary regulatory role of LKB1 for AMPK activation in chondrocytes. In these experiments, we saw increased NO release by $82 \%$ and $115 \%$ in response to IL- $1 \beta$ and TNF $\alpha$, respectively, compared with control cells (Figure $1 \mathrm{C}$ ). In addition, release of MMP-3 and MMP-13 in response to IL-1 $\beta$ and TNF $\alpha$ were also enhanced in LKB1 knockdown chondrocytes (Figure 1D,E). Conversely, overexpression of LKB1 in chondrocytes via transfection prevented dephosphorylation of AMPK $\alpha$ and inhibited NO production and MMP-3 and MMP-13 release in response to IL-1 $\beta$ and TNF $\alpha$ (data not shown).

\section{Loss of phosphorylation of LKB1 and AMPK $\alpha$ in cultured human knee osteoarthritic chondrocytes}

Since decreased phosphorylation of AMPK $\alpha$ was previously observed in human knee OA chondrocytes [13], we tested here for concurrent decrease of chondrocyte LKB1 and AMPK $\alpha$ phosphorylation. Cultured primary human chondrocytes (passage 1) with a range of normal 
A.

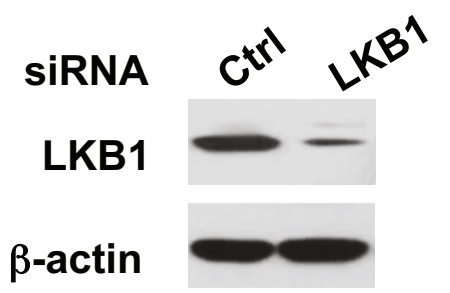

B.

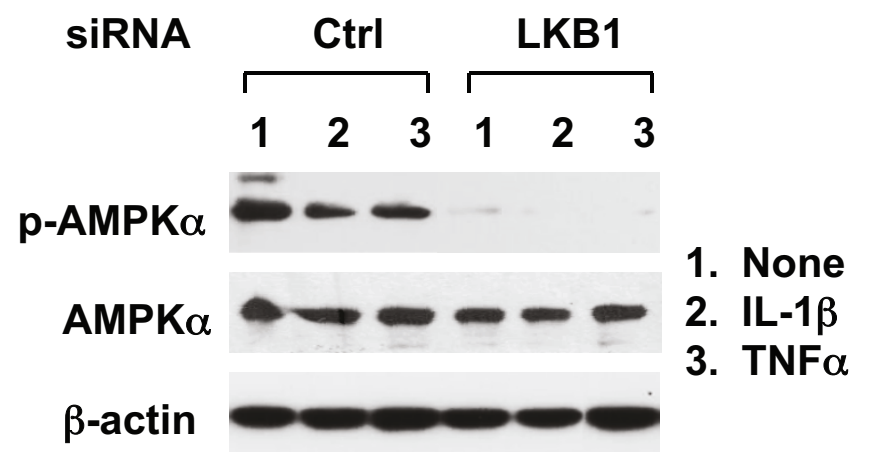

C.

NO Release

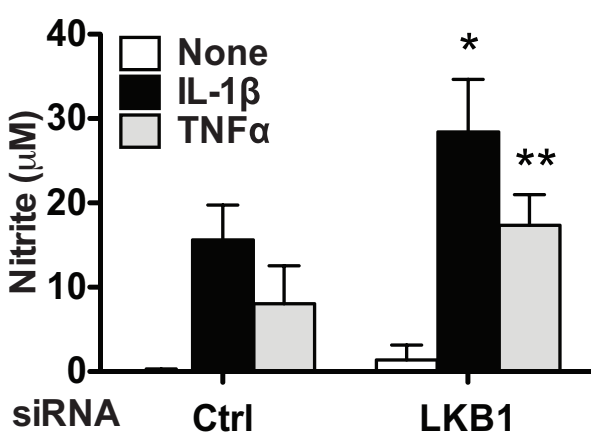

D.

MMP-3

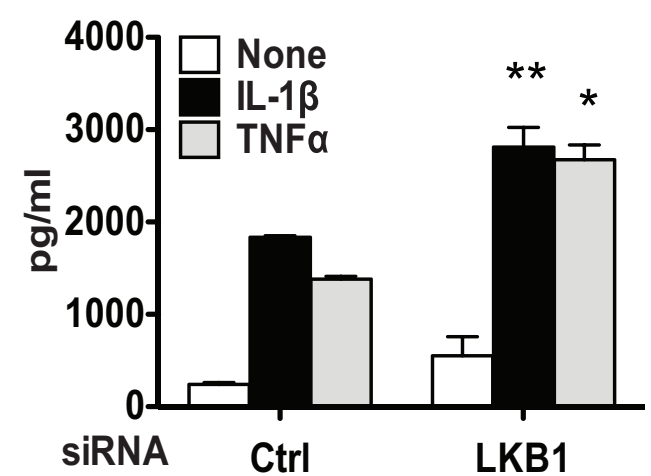

E.

MMP-13

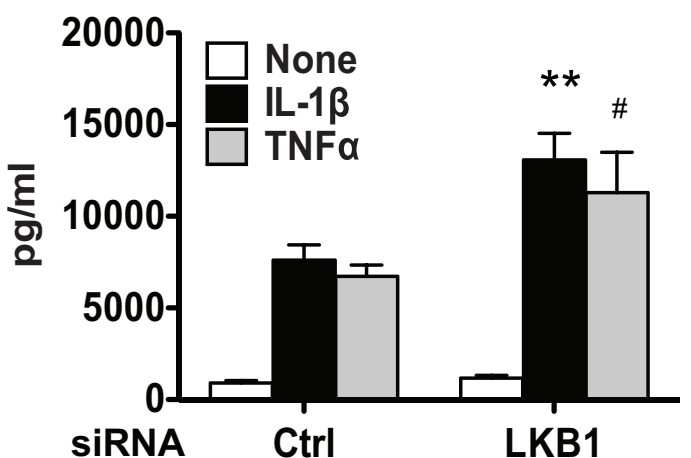

Figure 1 Knockdown of LKB1 in articular chondrocytes attenuates AMPK activity and promotes matrix catabolic responses. Human primary normal knee articular chondrocytes were transfected with liver kinase B1 (LKB1) and control siRNA. Two days after transfection, cells were either (A) used directly for western blot analysis of LKB1 expression or (B) treated with IL-1 $\beta$ (10 ng/ml) and TNF $\alpha$ (10 ng/ml) for 18 hours followed by western blot analysis for phosphorylation of AMP-activated protein kinase alpha (AMPK $\alpha$ ) and total AMPK $\alpha$. (C) Nitric Oxide (NO) release, (D) matrix metalloproteinase (MMP)-3 release and (E) MMP-13 release were then analyzed from the conditioned media by Griess reaction and ELISA, respectively. Data representative of three individual experiments. ${ }^{*} P<0.001,{ }^{* *} P<0.01, \# P<0.05$ relative to the control (Ctrl). 
(grade I), mild OA (grade II) and OA (grade III) were subjected to SDS-PAGE/western blot analysis of phosphorylation of LKB1 and AMPK $\alpha$. Phosphorylation of LKB1 was constitutively present in grade I and grade II chondrocytes, but was substantially decreased in grade III chondrocytes (Figure 2), where there was decrease in AMPK phosphorylation (Figure 2).

\section{Concomitant reduction of phosphorylation of LKB1 and} AMPK $\alpha$ in mouse knee osteoarthritic and aged mouse knee cartilages in situ

Immunohistochemistry analysis of mouse knee sections of surgically induced OA and sham control (with Osteoarthritis Research Society International scores of 5 and 0 , respectively) revealed that phosphorylated LKB1 and AMPK $\alpha$ were concomitantly decreased in mouse OA knee cartilage, compared with the sham control (Figure 3). Additionally, immunohistochemistry analysis of mouse knee sections 6, 12 and 24 months old (with Osteoarthritis Research Society International scores of 0.5, 1 and 1, respectively) revealed that the numbers of cells staining positively for either phosphorylated LKB1 and AMPK $\alpha$ were similar in 6-month-old and 12-month-old mouse knee cartilages, but were concomitantly decreased in 24-month-old mice knee cartilages (Figure 4).

\section{Decreased phosphorylation of LKB1 and AMPK $\alpha$ and} increased apoptosis and catabolic responses in chondrocytes after biomechanical injury in vitro We cultured normal bovine knee chondrocytes embedded in $2 \%$ alginate (three-dimensional) for 3 to 4 weeks to allow extracellular matrix production [18] before subjecting them to sub-lethal injury by continuous dynamic unconfined compression at $24 \%$ strain, $12 \%$ amplitude, $0.5 \mathrm{~Hz}$ for 16 hours as described in Materials and methods. The chondrocyte-alginate constructs were then cultured and collected at $0,1,2$ and 5 days after injury. As seen in Figure 5A, activation of caspase-3, an apoptosis mediator, became detectable in chondrocytes at 1, 2 and 5 days after compression. In contrast, phosphorylation of LKB1 and AMPK $\alpha$ started to decrease at 1 day and became attenuated at 5 days post injury (Figure $5 \mathrm{~A}$ ). Significant induction of NO release was seen immediately after injury and leveled off at 2 days post injury (Figure 5B). Similar results were observed for GAG release (Figure $5 \mathrm{C}$ ) that could reflect matrix catabolism. Induction of MMP-3 expression was seen at both mRNA and protein levels, with the highest level at 2 days after compression (Figure 5D,E). Taken together, decreased concurrent phosphorylation of LKB1 and AMPK $\alpha$ were associated with increased proapoptotic and catabolic responses in chondrocytes following mechanical injury.

Suppression of increased catabolic responses to injury by AMPK pharmacologic activators in cultured chondrocytes

Bovine knee chondrocytes embedded in alginate, which had first been cultured for 3 to 4 weeks, were pretreated with AMPK pharmacologic activators AICAR $(1 \mathrm{mM})$ and A-769662 (0.5 mM) for 24 hours before being subjected to biomechanical injury. After culture for 2 days post injury, the chondrocyte-alginate constructs and the conditioned media were collected for analysis of catabolic responses. As shown in Figure 6, both AICAR and A-769662 prevented dephosphorylation of AMPK $\alpha$ (Figure 6A) and inhibited caspase-3 activation (Figure 6B) after mechanical

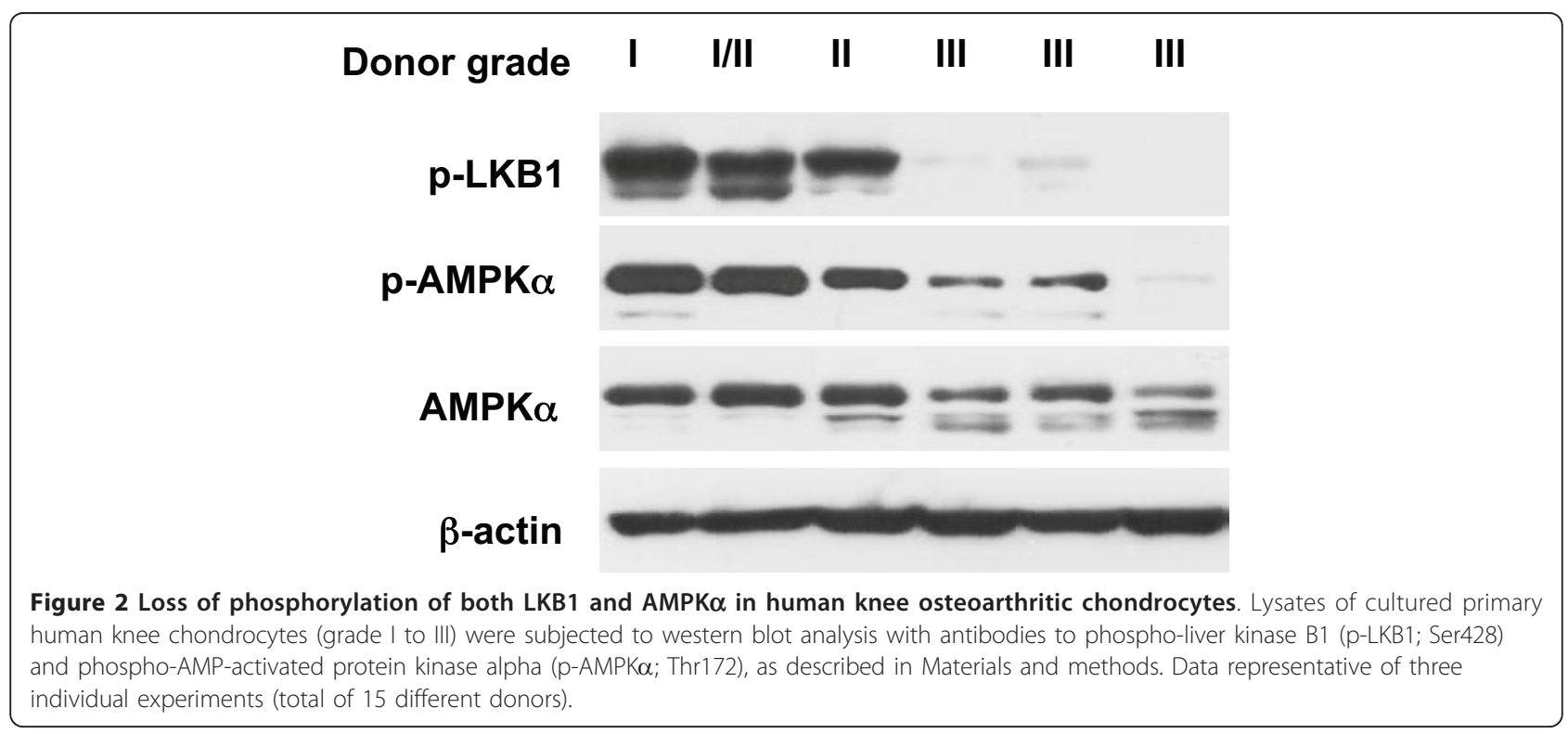




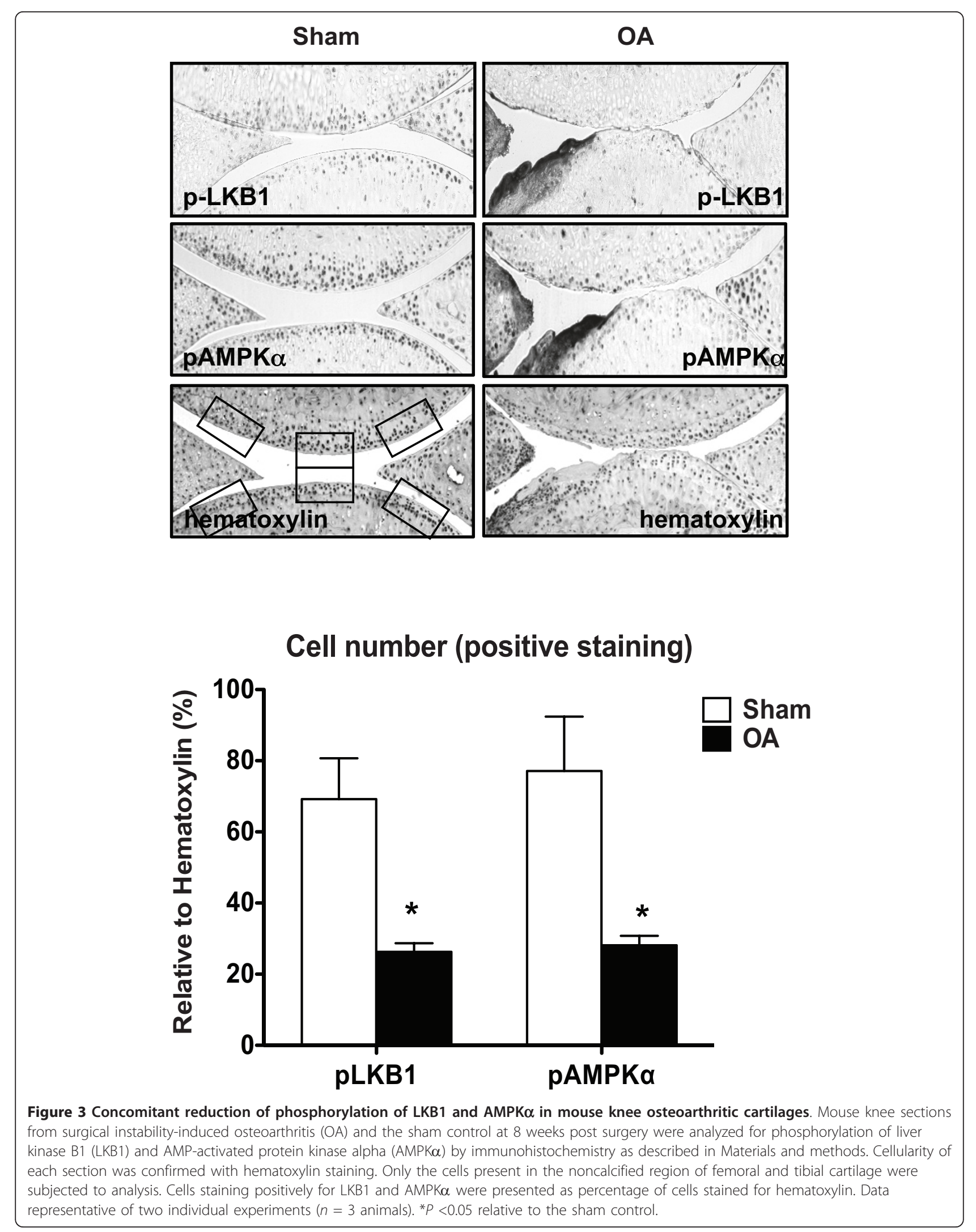




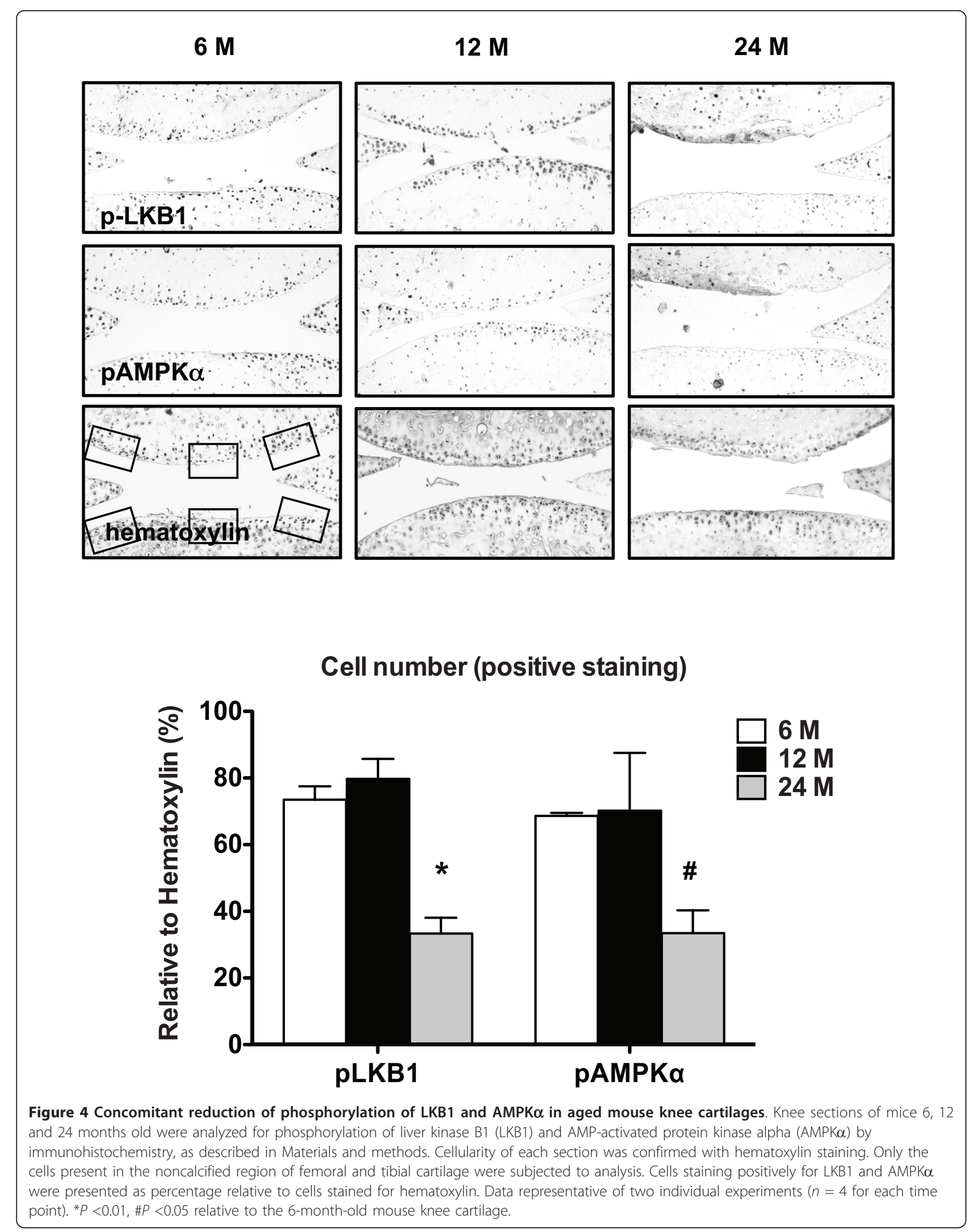


A.

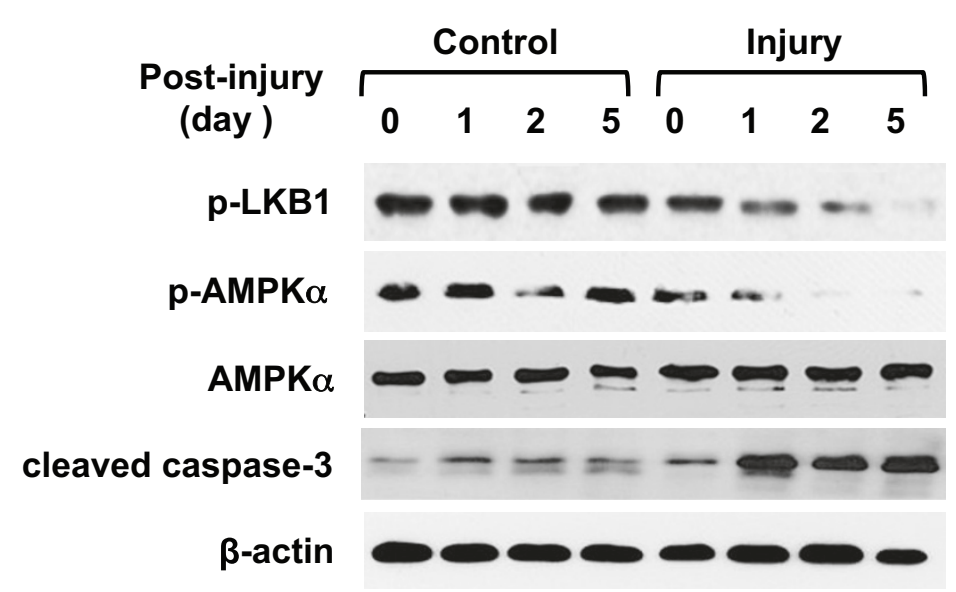

B.

\section{NO Release}

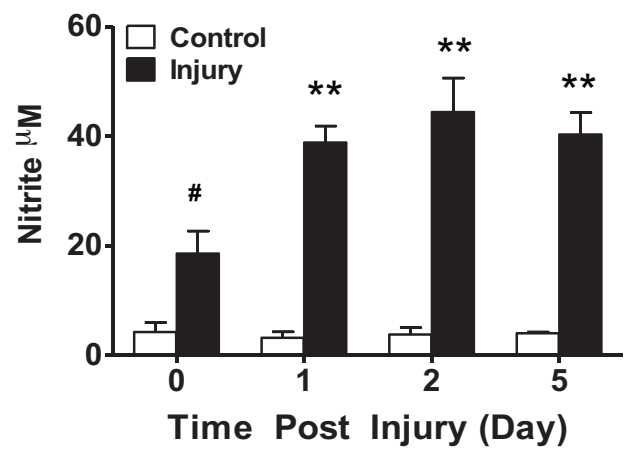

D.

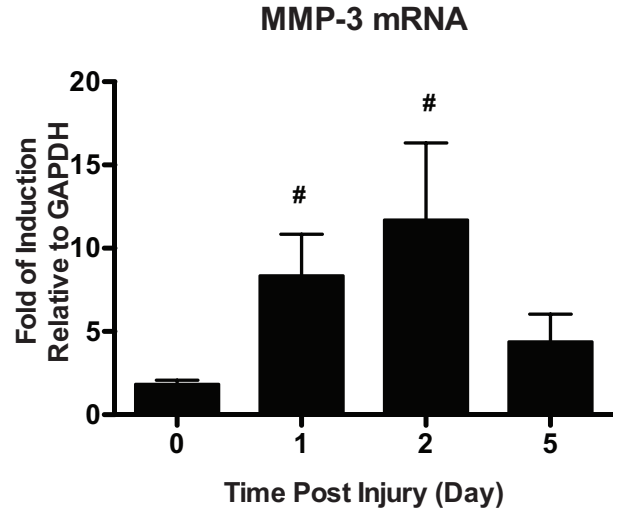

C.

\section{GAG Release}

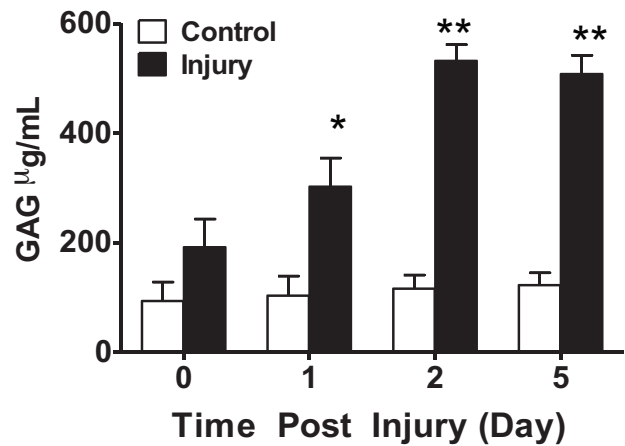

E.

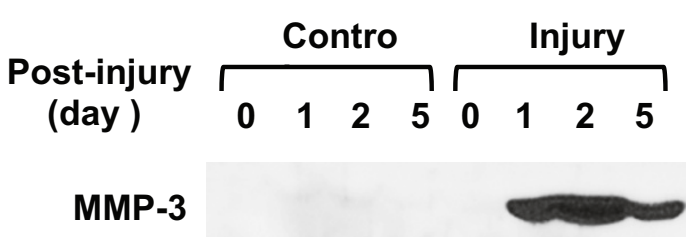

Conditioned media

Figure 5 Decreased LKB1 and AMPK $\alpha$ phosphorylation and increased apoptosis and catabolic responses after biomechanical injury. Loss of phosphorylation of liver kinase B1 (LKB1) and AMP-activated protein kinase alpha (AMPK $\alpha$ ), in addition to increased caspase-3 activation, nitric oxide (NO) release, and catabolic activities in bovine chondrocytes after biomechanical injury. Bovine knee articular chondrocytes were embedded in alginate disks and cultured for 3 to 4 weeks, followed by the sub-lethal biomechanical injury condition described in Materials and methods. The chondrocyte-alginate constructs were collected at 0, 1, 2 and 5 days after injury. (A) Chondrocytes from both injured and control groups were then released by dissolution of alginate, and subjected to western blot analysis for expression of cleaved caspase-3, phosphorylation of LKB1 and AMPK $\alpha$, and total AMPK $\alpha$. Conditioned media were employed for analysis of (B) NO and (C) glucosaminoglycan (GAG) release by Griess reaction and DMMB assay, respectively, and (E) matrix metalloproteinase (MMP)-3 release by western blot analysis. (D) MMP-3 mRNA expression was also examined by quantitative RT-PCR. Data representative of three individual experiments $(n=3$ replicates for each condition). ${ }^{* *} P<0.001$, ${ }^{*} P<0.01, \# P<0.05$ relative to the control. 
A.

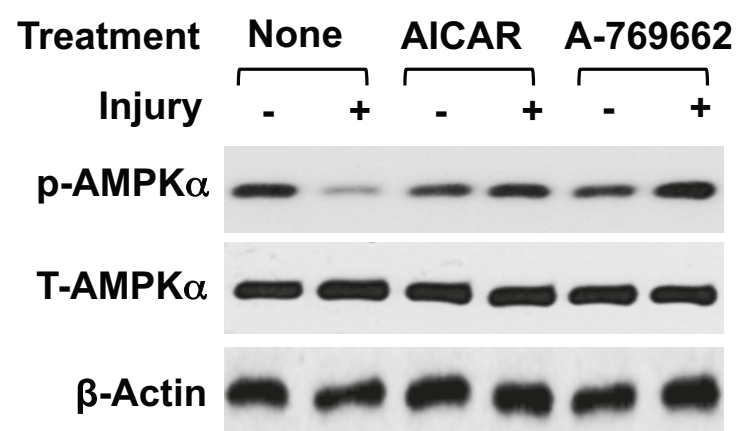

C.

Nitric Oxide Release

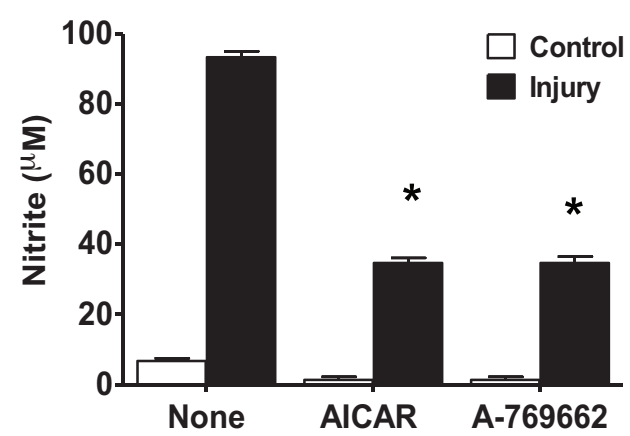

E.

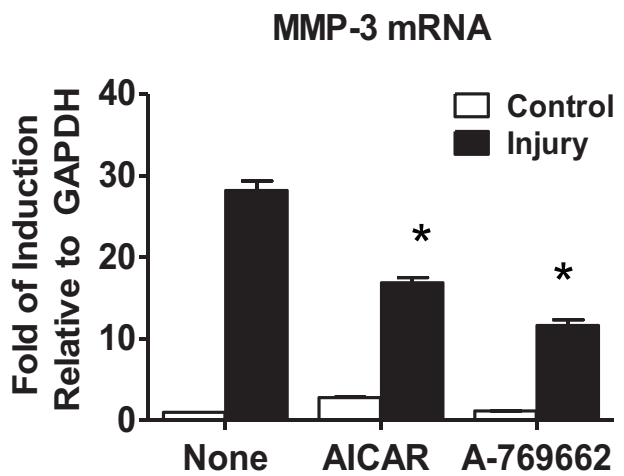

B.

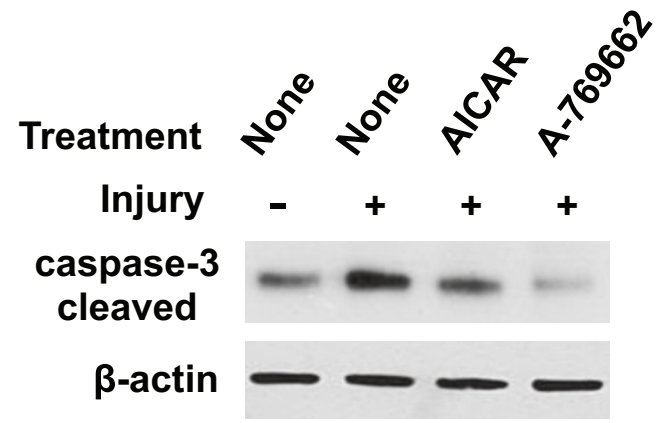

D. GAG Release

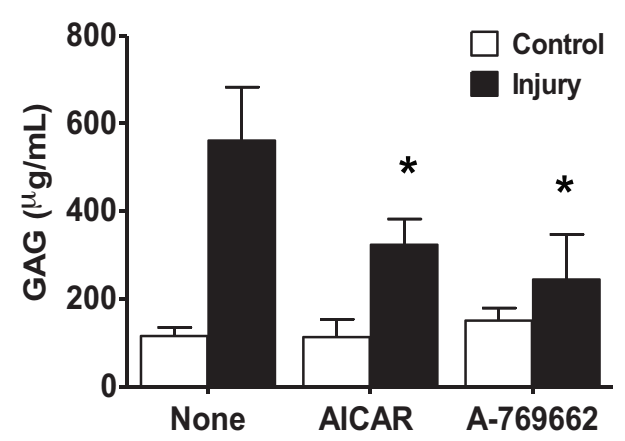

F.

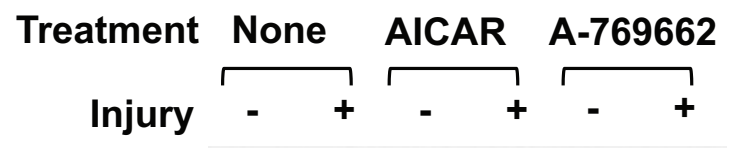

MMP-3

\section{Conditioned media}

Figure 6 AMPK pharmacologic activators inhibit catabolic responses of chondrocytes to biomechanical injury. Bovine chondrocytealginate disks were pretreated with 5-aminoimidazole-4-carboxyamide ribonucleoside (AICAR; 1 mM) and A-769662 (0.5 mM) for 24 hours, and then subjected to mechanical injury as described in Materials and methods. After culturing for 2 days, the chondrocytes released from alginate and the conditioned media were used for analysis of (A) phosphorylation of AMP-activated protein kinase alpha (AMPK $\alpha$ ) and total AMPK $\alpha$,

(B) cleaved caspase-3, (C) nitric oxide release and (D) glucosaminoglycan (GAG) release, and (E, F) matrix metalloproteinase (MMP)-3 expression. Data representative of two individual experiments ( $n=3$ replicates for each condition). ${ }^{*} P<0.01$ relative to the control. 
injury. In parallel, both AICAR and A-769662 dramatically inhibited NO and GAG release and MMP-3 expression at both mRNA and protein levels after mechanical injury (Figure 6C,D,E,F).

\section{Discussion}

This study demonstrated that activation of AMPK requires LKB1 in chondrocytes. Knockdown of LKB1 expression via siRNA resulted in attenuation of basal phosphorylation of $A M P K \alpha$, as well as significant enhancement of catabolic responses to IL-1 $\beta$ and TNF $\alpha$ in chondrocytes. Conversely, overexpression of LKB1 in chondrocytes via transfection prevented dephosphorylation of AMPK $\alpha$ and inhibited NO production and MMP-3 and MMP-13 release in response to IL-1 $\beta$ and TNFa (data not shown). We observed concomitant loss of phosphorylation of LKB1 and AMPK $\alpha$ in human knee OA chondrocytes (grade III) and in mouse knee OA cartilages.

Aging is a major risk factor for development of OA [1-4]. Decreased AMPK activation is linked with several age-associated diseases [19]. For example, deficiency of AMPK exacerbates aging-induced myocardial dysfunction [20]. Aging also impairs AMPK activation and suppresses insulin-stimulated glucose uptake into rat skeletal muscles, which is held to enhance the development of metabolic syndrome [21]. Here, we observed that phosphorylation of both LKB1 and AMPK $\alpha$ were present at 6 and 12 months of age in mouse knee cartilages, but were reduced significantly in 24-month-old mouse knee cartilages. Our results suggest that decreased capacity for linked LKB1 and AMPK activation in aging chondrocytes may increase susceptibility to OA development.

Biomechanical injury can promote the development and progression of OA $[7,22]$. Our translational studies of the pharmacologic AMPK activators AICAR and highly AMPK-selective A-769662 suggested that maintaining AMPK activity in injured chondrocytes can promote preservation of cartilage matrix integrity. Significantly, we established and validated a new, sub-lethal biomechanical injury condition for chondrocytes in three-dimensional chondrocyte-alginate constructs. In this system, concurrent loss of phosphorylation of LKB1 and AMPK $\alpha$, associated with induction of caspase- 3 activation, release of NO and GAG, and MMP-3 expression, developed in response to injury. Conversely, treatment of chondrocytealginate constructs with AMPK pharmacologic activators AICAR and A-769662 prevented dephosphorylation of AMPK $\alpha$ and inhibited caspase- 3 activation and catabolic responses induced by biomechanical injury.

There are several core limitations to this study. First, chondrocytes can retain their phenotype and produce physiological cartilage matrix components when immobilized in alginate, but the biomechanical properties of three-dimensional chondrocyte-alginate constructs may not be identical to those in articular cartilage. Future biomechanical injury studies with articular cartilage explants would be of interest. Second, the scope of analyses herein focused on the relationship between LKB1 and AMPK activity. However, regulation of AMPK involves phosphorylation by two upstream kinases in addition to LKB1; that is, calcium calmodulin-dependent kinase kinase beta and transforming growth factor betaactivated kinase $1[10,11]$. Whether calcium calmodulindependent kinase kinase beta and transforming growth factor beta-activated kinase 1 regulate AMPK activation in chondrocytes remains to be determined. Protein phosphatases such as protein phosphatase $2 \mathrm{~A}$ and protein phosphatase $2 \mathrm{C}$ negatively regulate AMPK activity through dephosphorylation of AMPK $\alpha[10,11]$. Unlike the case for LKB1, however, we have not observed any significant differences in expression of protein phosphatase 2C $\alpha$ between human knee OA (grade III) and normal chondrocytes (grade I) (R. Liu-Bryan, unpublished observation). Third, AMPK promotes autophagy [23,24], which may be a protective mechanism against injury in chondrocytes that decreases in aging cartilage $[16,25]$. Analysis of the relationships between LKB1 and AMPK activity and autophagy in aging cartilage and biomechanically injured chondrocytes were beyond the scope of this study, but would be of interest.

\section{Conclusion}

Reduction in functionally linked LKB1 and AMPK activation in chondrocytes associated with aging, inflammation and biomechanical injury has the potential to disrupt cartilage extracellular matrix homeostasis, and thereby could contribute to OA development and progression. Pharmacologic AMPK activation is a novel potential therapeutic approach for OA that merits further investigation.

\section{Abbreviations}

AICAR: 5-aminoimidazole-4-carboxyamide ribonucleoside; AMPK: AMPactivated protein kinase; DMEM: Dulbecco's modified Eagle's medium; ELISA: enzyme-linked immunosorbent assay; FCS: fetal calf serum; GAG:

glucosaminoglycan; IL: interleukin; LKB1: liver kinase B1; MMP: matrix metalloproteinase; NO: nitric oxide; NF: nuclear factor; OA: osteoarthritis; PBS: phosphate-buffered saline; PCR: polymerase chain reaction; RT: reverse transcriptase; TNF: tumor necrosis factor.

\section{Competing interests}

RL-B who holds a patent (filed through University of California, San Diego, CA, USA) that partially related to the content of the manuscript; however, $\mathrm{RL}-\mathrm{B}$ has not received any reimbursements, fees, funding, or salary from this organization that holds the patent relating to the content of the manuscript. The remaining authors declare that they have no competing interests.

\section{Authors' contributions}

RL-B and RT had full access to all data in the study and take responsibility for the integrity of the data and accuracy of the data analysis. FP and RL-B were responsible for acquisition of data. $F P, M H, R J, M L, R T$, and $R L-B$ were 
responsible for study conception and design, and for analysis and interpretation of data. All authors were involved in drafting or revising the manuscript critically for important intellectual content, and all authors approved the final version to be published.

\section{Acknowledgements}

Studies were supported by the Research Service of the Department of Veterans Affairs, National Institutes of Health grants PO1 AG007996 (to ML and RT), AR54135 (to RT), HL077360 (to RT) and AR1067966 (to R-LB).

\section{Authors' details}

'VA San Diego Healthcare System, Department of Medicine, University of California San Diego, 111K, 3350 La Jolla Village Drive, San Diego, CA 92161, USA. ${ }^{2}$ Department of Medicine, The Ohio State University, 480 Medical Center Drive, Columbus, OH 43210, USA. ${ }^{3}$ Mechanical and Industrial Engineering Department, Montana State University, PO Box 173800 Bozeman, MT 59717-3800, USA. ${ }^{4}$ The Scripps Research Institute, 10550 North Torrey Pines Road, La Jolla, CA 92037, USA.

Received: 31 October 2012 Revised: 18 March 2013

Accepted: 25 July 2013 Published: 25 July 2013

\section{References}

1. Loeser RF, Goldring SR, Scanzello CR, Goldring MB: Osteoarthritis: a disease of the joint as an organ. Arthritis Rheum 2012, 64:1697-1707.

2. Goldring MB, Goldring SR: Osteoarthritis. J Cell Physiol 2007, 213:626-634.

3. Anderson DD, Chubinskaya S, Guilak F, Martin JA, Oegema TR, Olson SA, Buckwalter JA: Post-traumatic osteoarthritis: improved understanding and opportunities for early intervention. J Orthop Res 2011, 29:802-809.

4. Lotz M, Loeser RF: Effects of aging on articular cartilage homeostasis. Bone 2012, 51:241-248

5. Loeser RF: Aging and osteoarthritis. Curr Opin Rheumatol 2011, 23:492-496.

6. Griffin TM, Guilak F: The role of mechanical loading in the onset and progression of osteoarthritis. Exerc Sport Sci Rev 2005, 33:195-200.

7. Buckwalter JA, Martin JA, Brown TD: Perspectives on chondrocyte mechanobiology and osteoarthritis. Biorheology 2006, 43:603-609.

8. Leong DJ, Hardin JA, Cobelli NJ, Sun HB: Mechanotransduction and cartilage integrity. Ann N Y Acad Sci 2011, 1240:32-37.

9. Leong DJ, Sun HB: Events in articular chondrocytes with aging. Curr Osteoporos Rep 2011, 9:196-201.

10. Steinberg GR, Kemp BE: AMPK in health and disease. Physiol Rev 2009, 89:1025-1078.

11. Witczak CA, Sharoff CG, Goodyear L: AMP-activated protein kinase in skeletal muscle: from structure and localization to its role as a master regulator of cellular metabolism. Cell Mol Life Sci 2008, 65:3737-3755.

12. Salminen A, Hyttinen JM, Kaarniranta K: AMP-activated protein kinase inhibits NF-KB signaling and inflammation: impact on health span and lifespan. J Mol Med 2011, 89:667-676.

13. Terkeltaub R, Yang B, Lotz M, Liu-Bryan R: Chondrocyte AMP-activated protein kinase activity suppresses matrix degradation responses to inflammatory cytokines IL-1 $\beta$ and TNFa. Arthritis Rheum 2011, 63:1928-1937.

14. Uhl M, Allmann KH, Ihling C, Hauer MP, Conca W, Langer M: Cartilage destruction in small joints by rheumatoid arthritis: assessment of fatsuppressed three-dimensional gradient-echo MR pulse sequences in vitro. Skeletal Radiol 1998, 27:677-682.

15. Grogan SP, Miyaki S, Asahara H, D'Lima DD, Lotz MK: Mesenchymal progenitor cell markers in human articular cartilage: normal distribution and changes in osteoarthritis. Arthritis Res Ther 2009, 11:R85.

16. Carames B, Taniguchi N, Otsuki S, Blanco FJ, Lotz M: Autophagy is a protective mechanism in normal cartilage, and its aging-related loss is linked with cell death and osteoarthritis. Arthritis Rheum 2010, 62:791-801.

17. Liu R, Liote F, Rose DM, Merz D, Terkeltaub R: Proline-rich tyrosine kinase 2 and Src kinase signaling transduce monosodium urate crystal-induced nitric oxide production and matrix metalloproteinase 3 expression in chondrocytes. Arthritis Rheum 2004, 50:247.

18. De Ceuninck F, Lesur C, Pastoureau P, Caliez A, Sabatini M: Culture of chondrocytes in alginate beads. Methods Mol Med 2004, 100:15-22.

19. Salminen A, Kaarniranta K: AMP-activated protein kinase (AMPK) controls the aging process via an integrated signaling network. Ageing Res Rev 2012, 11:230-241.
20. Turdi S, Fan X, Li J, Zhao J, Huff AF, Du M, Ren J: AMP-activated protein kinase deficiency exacerbates aging-induced myocardial contractile dysfunction. Aging Cell 2010, 9:592-606.

21. Qiang W, Weiqiang K, Qing Z, Pengju Z, Yi L: Aging impairs insulinstimulated glucose uptake in rat skeletal muscle via suppressing AMPKa. Exp Mol Med 2007, 39:535-543.

22. Buckwalter JA, Martin JA: Osteoarthritis. Adv Drug Deliv Rev 2006, 58:150-167.

23. Bohensky J, Leshinsky S, Srinivas V, Shapiro IM: Chondrocyte autophagy is stimulated by HIF-1 dependent AMPK activation and mTOR suppression. Pediatr Nephrol 2010, 25:633-642.

24. Bohensky J, Shapiro IM: Autophagy: a new phase in the maturation of growth plate chondrocytes is regulated by HIF, mTOR and AMP kinase. Cells Tissues Organs 2009, 189:88-92.

25. Carames B, Taniguchi N, Seino D, Blanco FJ, D'Lima D, Lotz M: Mechanical injury suppresses autophagy regulators and pharmacologic activation of autophagy results in chondroprotection. Arthritis Rheum 2012, 64:1182-1192.

doi:10.1186/ar4254

Cite this article as: Petursson et al:: Linked decreases in liver kinase B1 and AMP-activated protein kinase activity modulate matrix catabolic responses to biomechanical injury in chondrocytes. Arthritis Research \& Therapy 2013 15:R77.

\section{Submit your next manuscript to BioMed Central and take full advantage of:}

- Convenient online submission

- Thorough peer review

- No space constraints or color figure charges

- Immediate publication on acceptance

- Inclusion in PubMed, CAS, Scopus and Google Scholar

- Research which is freely available for redistribution

Submit your manuscript at www.biomedcentral.com/submit
C Biomed Central 